\title{
A Multi-Wideband Planar Monopole Antenna for 4G Devices
}

\author{
A. Asghar, M. Malick, M. Karlsson, Member, IEEE, and A. Hussain
}

\begin{abstract}
This paper presents a compact multiband planar antenna designed for mobile phone applications. The antenna performance is achieved by designing a planar monopole antenna into distributed radiating elements. The proposed antenna is comprised of a chopped circular radiator appended with a meander line and an L-strip coupled element which is an extension of the ground plane. The combination of a chopped circular patch and L-shaped coupling strip residing on the top side generates lower band while upper band resonances are attained separately by chopped circular resonator and meander line elements. The antenna shows a planar structure which occupies an area of $17.6 \times 56 \mathrm{~mm}$ and can be directly printed onto a circuit board at low cost making it especially suitable for mobile phone applications. The manufactured antenna is experimentally verified and covers several wireless communication bands, namely, LTE 750, GSM 850, GSM 900, DCS, UMTS-2110, Bluetooth, WLAN, WiMAX and UWB. The high frequency structure simulation (HFSS) is employed to design and analyze the antenna performance, and a practical structure was fabricated and tested. The measured and simulated return loss show good agreement.
\end{abstract}

Index Terms-multiband planar antenna, meander line, communication bands, mobile phone.

\section{INTRODUCTION}

$\mathrm{T}$ he remarkable progress in mobile communication systems has proportionally led to the advancement of antenna systems in the recent decade. The typical mobile communication system has rapidly evolved, emerging from analog systems to systems fully capable of multimedia transmission. On the other hand the rapid evolution has also come forward as an advancement in hand held mobile devices. Besides keeping the appearances of these devices attractive, devices must also be small in dimension i.e., not only in the plane parallel to the screen but also in the thickness to be competitive in the market. Attractiveness in appearance had greatly influenced prevailing internal antenna over external antenna, which are completely concealed in a mobile phone [1], [2]. There has been a huge trend of equipping the mobile devices with more components adding more functionality while reducing the size and thickness. Thus size of mobile devices has been consistently decreasing, making it slimmer and smaller with respect to space for an antenna, which is making a bit more challenging for antenna designers to meet such limitations.

Current trend in multi-band antenna designs used in mobile devices can be categorized into three types: slot-type antennas, monopole antennas and planar inverted-F antennas (PIFA) [3], [4]. Slot antenna can also be operated as a quarter-wavelength resonant structure, when a slot is cut at the edge of ground plane [5]-[7]. Multiple resonant modes are achieved through different slots of various geometric cuts onto the radiator and ground plane [8]-[10]. In [11]-[13], antenna size is decreased and its bandwidth is increased through employing capacitive and inductive loading/de-loading techniques. Also implementation of parallel combination of slot and PIFA on handset antenna designs can be seen in [14], [15]. The third group, monopole antennas are also operated as a quarterwavelength resonant structures [16], [17]. The planar monopole has a capability to provide a wide impedance bandwidth. There are several techniques which facilitate multiband operation. In [18], by adding some branches and introducing fractal patterns to the monopole design, multiple resonant bands are achieved. Another interesting work is implemented by a two branch monopole on a dielectric loading to minimize the antenna size as shown in [19].

In this article, a planar multiband antenna is proposed for mobile communication \& ultra wideband applications. The antenna performance is achieved by a planar monopole antenna with multi-resonating elements. The multiband antenna covers all popular cellular and internet communication bands: LTE 750, GSM 850 (824-960 MHz), GSM 900 (880-960 MHz), DCS (1710-1880 MHz), UMTS2110 (2110-2200 MHz), 2.4 GHz WLAN (2400-2480 MHz), ISM/Bluetooth (2400-2480 MHz), WiMAX (2500-2690 MHz \& 3400-3600 MHz) and Ultra Wideband (3.1-10.6 GHz). This design is fully planar, fairly compact, and utilizes low cost Roger 4360 substrate material.

\section{ANTENNA CONFIGURATION}

Figure 1 shows the geometry of the proposed planar monopole antenna, which is fabricated using a $0.305 \mathrm{~mm}$ thick, $48 \mathrm{~mm}$ wide, and $119.6 \mathrm{~mm}$ high Rogers 4360 substrate having a dielectric constant of 6.15 . On the rear side of dielectric substrate, a $47 \times 102 \mathrm{~mm}$ ground plane resides. As depicted in Figure 1(a), the antenna element is designed on the top frontal portion of dielectric substrate covering an area of $43.1 \times 13.5$ mm which is conventionally referred to as the antenna area. The proposed antenna can be classified into three major resonant structures according to its inherent functionality: a main radiator (a chopped circular radiator), a meander line element, and a parasitic element (L-strip resonator). All these elements are printed coplanar with the ground plane.

The geometry of the main radiator is illustrated in Figure 1(b). It is composed of a semicircular shape with a radius of $29 \mathrm{~mm}$. Its left and right portion is chopped and a meander line is introduced on the right side. The extended meander element is 
$1 \mathrm{~mm}$ thick with a dimension of $12 \mathrm{~mm}$ in width and $10 \mathrm{~mm}$ in height. It consists of four turns with a total length of $48 \mathrm{~mm}$. Thus the main radiator with a meander line has an area of 13.5 $\times 43.1 \mathrm{~mm}$. The radiator and microstrip feed line is printed on the same side of the dielectric substrate. The feed line of $50 \Omega$ was realized, using standard design formulas [20]. The feed line length, width and feed gap is $15 \mathrm{~mm}, 0.42 \mathrm{~mm}$ and 0.6 $\mathrm{mm}$, respectively. The feed line is connected to an SMA connector through a hole in the substrate and ground plane.

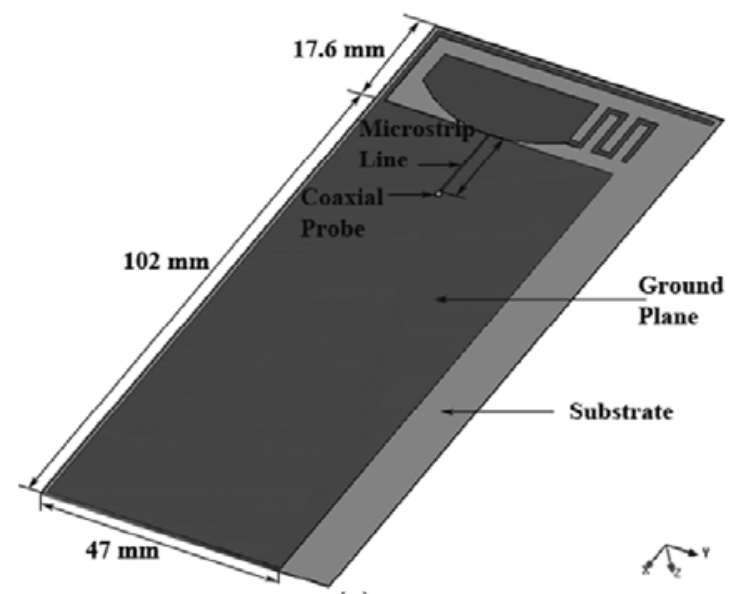

(a)

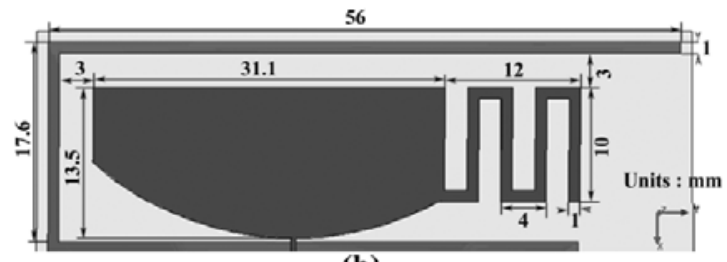

(b)

Figure 1: Configuration of proposed wideband planar monopole antenna, (a) perspective view of entire structure, (b) Geometry and dimensions of proposed antenna.

The parasitic structure lies in the top-left corner of the antenna area and on the same side as the ground plane; it is comprised of an L-strip of $1 \mathrm{~mm}$ in width and of $73.6 \mathrm{~mm}$ in length which is directly fed from the ground plane.

\section{ANTENNA DESIGN: CONCEPT \& PROCEDURES}

This paper is primarily concerned with the designing of a miniaturized antenna that is capable to cover standard mobilephone communication bands as well as the UWB. This requires integrating several communication bands in a single antenna design while maintaining high efficiency. Throughout literature, interesting multiband antennas for mobile applications have been discussed [21], [22]. Several resonance frequencies can be achieved, but through combination of more than one resonating elements, to cover all of the bands. Recent literature has also presented improved designs covering multiple bands for mobile applications [23], [24].

It is essential that the aforementioned design approaches should be taken in account for achieving an enhancement in impedance bandwidth as well as reduction of antenna size of the conventional monopole antenna. A novel design is formulated here, through combination of L-strip and chopped circular monopole antenna with meander line. Simulations were carried out using version 12 of HFSS.

\section{A. Preliminary Design of Main Radiator}

The design of main radiator is comprised of a chopped circular monopole, ultimately reducing the size of the antenna. The dimension of the "Type I" is $45 \mathrm{~mm}$ wide and $13.5 \mathrm{~mm}$ in height and it is depicted in Figure 2(a). In summary, the chopping of the main radiator is done in a precise manner to provide a good coupling with the ground which results in producing a good impedance bandwidth.

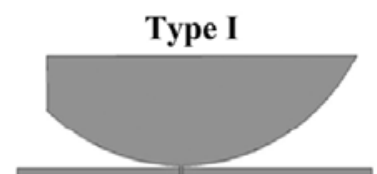

(a) (c)

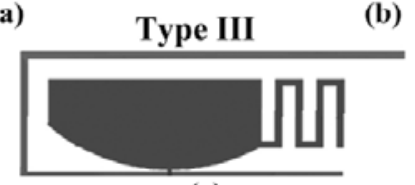

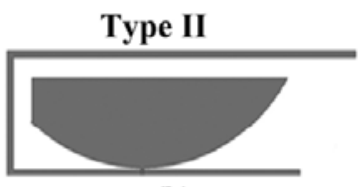

(b)
Figure 2: Design configuration of different variant of proposed antenna (a) Type 1, (b) Type 2, (c) Type 3 antennas

Apart from the design procedure, the simulation showed that impedance bandwidth acquired from "Type I" encompasses the whole UWB band, as well as some higher frequency bands of WWAN. The simulated result of return loss in Figure 3 with dot line depicts a span from 2.3-11 GHz with VSWR less than 2 which is quite good for a mobile phone antenna [25].

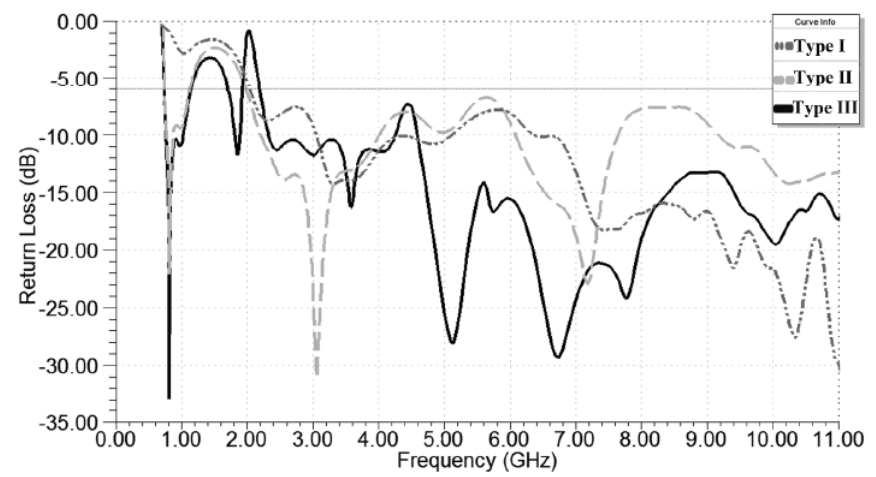

Figure 3: Comparison of simulated return loss for the type 1, 2, and 3 antennas.

\section{B. Design of Parasitic Structure}

The achieved wide frequency band from the "Type I" antenna fails to cover all of the desired frequency bands; operating bands such as LTE and GSM 850/900 MHz are missing. The remaining lower frequency bands of WWAN are achieved through inclusion of L-strip parasitic element in the antenna, which is appended to the upper-left corner of the ground. The modified antenna is termed as "Type II" antenna as shown in Figure 2(b). The performance of the antenna is determined by the geometrical parameter of L-strip i.e., its length. L-strip enhancement technique is instrumental in attaining lower frequency band [26]. It serves to modify the current distribution on the radiator and the ground plane which 
changes the input impedance and consequently results in enhancing the impedance bandwidth. The following analytical formula (1) is used to determine the optimal length of the inverted L-strip structure for the desired resonating frequency [27]:

$$
f_{\text {res }}=\frac{\mathrm{c}}{4 \sqrt{\varepsilon_{e f f}}\left(L_{V}+L_{H}\right)}
$$

Where, $f_{\text {res }}$ is resonating frequency, c is speed of light in vacuum and $\varepsilon_{\text {eff }}$ is effective dielectric constant. With the length of the parasitic structure's vertical section $L_{V}$ fixed at $17.6 \mathrm{~mm}$, the resonant mode excited through the parasitic structure is controlled by varying the length of the horizontal section $L_{H}$. In our case we adjusted the length of the horizontal section to $56 \mathrm{~mm}$ to achieve the required resonating mode at $800 \mathrm{MHz}$. An optimal behavior of L-strip for this modified design, for achieving an impedance bandwidth in the lower frequency mode, principally depends upon the coupling in between the L-strip and main radiator structure. A spacing of 3 $\mathrm{mm}$ is defined between both resonating structures. This modification in design further enables the coverage of LTE $750 \mathrm{MHz}$ and GSM 850/900 $\mathrm{MHz}$ as illustrated in figure 3 with dashed line.

\section{Final Design}

Traversing through the "Type I" \& “Type II” is carried out through modifications in the basic design in a stepwise manner to achieve the remaining desired operating bands. Still an important band of DCS was required to be covered. Meander line technique is used here as a potential solution to obtain this band. This solution is introduced through addition of a meander line on the right side of the main radiator. The length of the meander line is formulated using (1). Introduction of meander line enabled the antenna to cover the frequency band of DCS (1710-1880 MHz).

\section{TOTAL RADIATION EFFICIENCY MEASUREMENT IN REVERBERATION CHAMBER}

The designed antenna is fabricated and measured for total radiation efficiency in a reverberation chamber [29] - [30]. Reverberation chamber has been well accepted over-the air (OTA) measurement tool for measuring both active and passive devices and emulates rich isotropic multipath (RIMP) environment. This environment is proposed as a reference OTA test environment for measurement of wireless mobile devices such as mobile phones working in a rich scattering environment to emulate real-life fading i.e. Rayleigh fading [31]. The fabricated antenna is designed for use in mobile communication and therefore measurement in reverberation chamber suited best for it. The measurement uncertainty in reverberation chamber is well within the acceptable range of $\pm 0.5 \mathrm{~dB}$ [32]. The measurements done in reverberation chamber are repeatable therefore these measurements can be easily repeated with same accuracy in the measured results. The reverberation chamber is a metallic cavity which has several stirrers to stir the energy. The device-under-test (DUT) resides on a rotating platform stirrer and observes Rayleigh fading environment during the measurement sequence.
The path-loss inside the reverberation chamber, cable losses, and internal losses of the antenna are measured and calibrated out. The path-loss inside the reverberation chamber is measured with vector network analyzer using high efficiency reference antenna present inside the chamber. This reference antenna is wideband, dual-polarized, and has nearly isotropic radiation pattern. After the reference measurement, the antenna-under-test (AUT) is placed on the rotating platform inside the reverberation chamber and observes the same Rayleigh fading environment to measure its radiation efficiency.

\section{RESUltS \& DISCUSSION}

As per the parameters mentioned in section II, an antenna prototype is fabricated and measured. Figure 4 shows the measured and simulated return loss for the fabricated antenna prototype. The characteristics of the proposed antenna have been simulated by using the full wave electromagnetic simulation tool HFSS. Return loss of the proposed prototype is measured using vector network analyzer.

Generally, a 6-dB return loss is acceptable for mobile phone applications [25]. The measured result shows that the proposed antenna achieves multiple wide frequency bands within an acceptable return loss range. The result is mainly divided into lower and higher frequency bands respectively. The lower frequency band exhibits a resonance at $820 \mathrm{MHz}$ and has a bandwidth of $240 \mathrm{MHz}(740-980 \mathrm{MHz})$ with a 3:1 VSWR; it mainly covers LTE $750 \mathrm{MHz}$ and GSM 850/900 $\mathrm{MHz}$ operational bands. The upper band comprises of multiple wide frequency bands, the first band achieves a bandwidth of $750 \mathrm{MHz}(1200-1900 \mathrm{MHz})$ with a 3:1 VSWR. This band mainly provides the operation of GPS \& DCS. Simulation result exhibits a lesser bandwidth than the measured results excluding the GPS band. In addition to above achieved operational bands; the measured results also covers an additional band of UMTS-2110.

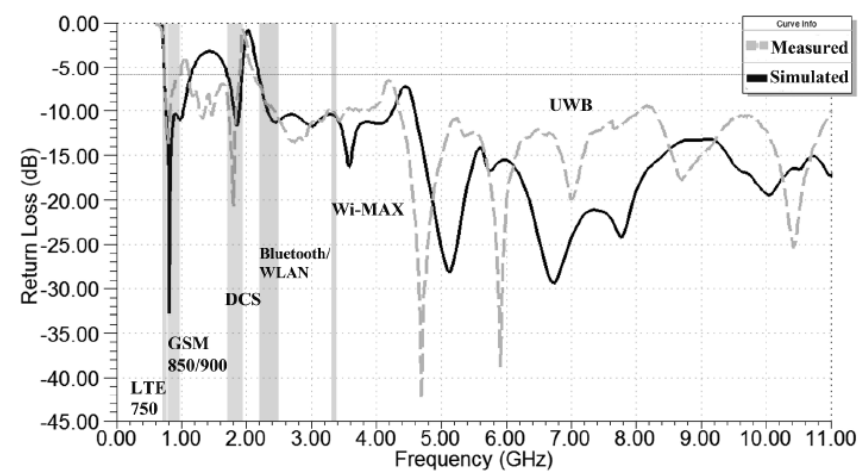

Figure 4: Simulated and measured return loss of the proposed antenna.

The second band is an extremely wideband, having a span of 8.7 GHz (2.3-11 GHz) with a 2:1 VSWR. An exception of approximately $300 \mathrm{MHz}$ band (4.0-4.3 GHz) exists with a 2.5:1 VSWR. This band covers a wide range of wireless communication standards i.e., Bluetooth, WLAN, WiMAX, and UWB. 
A small discrepancy in terms of frequency shift is observed between the simulated and measured return loss of the proposed antenna. These frequency shifts are attributed to the manufacturing, tolerance errors, surface roughness, soldering, and also due to the connector's effect. This association of the antenna on the PCB resulted in small shifts of resonances without any noticeable degradation of their bandwidths. This kind of behavior is also reported in [28].

The excited surface current distributions are observed at different frequencies of interest. The radiating element of the proposed antenna is excited at $820 \mathrm{MHz}$ and $1820 \mathrm{MHz}$ respectively as presented in figure 5 .

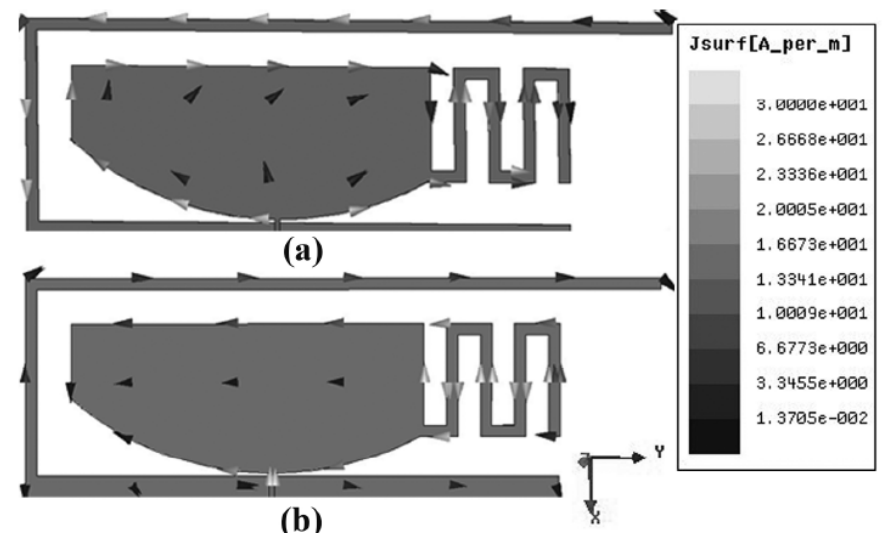

Figure 5: Simulated results of the surface current distribution on the radiating patch at (a) $820 \mathrm{MHz}$ (b) $1820 \mathrm{MHz}$.

At $820 \mathrm{MHz}$ excitation, a larger surface current distribution is observed for the longer path along the L-strip resonator illustrated in figure 5(a). This implies that the L-strip is the dominating radiating element of the proposed antenna at 820 $\mathrm{MHz}$; more or less the L-strip operates as a quarterwavelength monopole structure. For the $1820 \mathrm{MHz}$ operation, majority of the surface current flows on the meander line which is evident in figure 5(b), behaving as a quarterwavelength structure. In conclusion, the current distribution on the antenna at lower frequency bands is largely at the L-strip and meander line operating as a quarter-wavelength structure. The fabricated prototype was mounted in reverberation chamber in the Antenna Lab at Chalmers University of Technology to measure total radiation efficiency. The measured antenna efficiency of the prototype is plotted over the frequency range of 0.5 to $8 \mathrm{GHz}$ as depicted in figure 6 . The antenna efficiency in the lower and upper operating bands is above $-2.3 \mathrm{~dB}$, rendering the designed antenna suitable for practical applications.

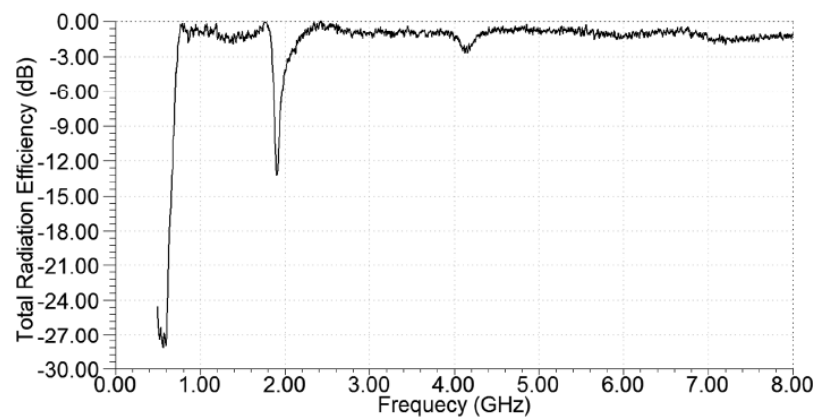

Figure 6: Measured antenna total radiation efficiency.
The Simulated radiation pattern of the proposed antenna is illustrated in figure 7. Both $E_{\theta}$ and $E_{\varphi}$ components are observed in three planes i.e., $x-y, x-z$ and $y-z$ plane at 0.82 $\mathrm{GHz}$ and $6 \mathrm{GHz}$ respectively. The radiation plot of the antenna exhibits a nearly omnidirectional pattern.

\section{CONCLUSION}

A compact planar monopole antenna for mobile phone achieving a wide impedance bandwidth has been presented. The antenna was initially designed as a chopped circular monopole and subsequently modified in the design process, firstly interfaced to couple with an L-strip parasitic resonant element and later appended with meander line. Wide impedance bandwidth is achieved by means of multiple resonating elements interacting with each other to attain multiresonance bands. The proposed antenna design is relatively compact in size while it covers a wide range of important frequency bands i.e., LTE $750 \mathrm{MHz}$, GSM 850/900 MHz, DCS, UMTS-2110, Bluetooth, WLAN, WiMAX \& UWB application. It exhibits nearly an omnidirectional radiation pattern. Measured VSWR of the proposed antenna is less than 2 for the UWB while frequency band $3.1-10.6 \mathrm{GHz}$ where it is less than 3 . The aforementioned attributes make it particularly attractive for portable devices such as smart phones and PDAs

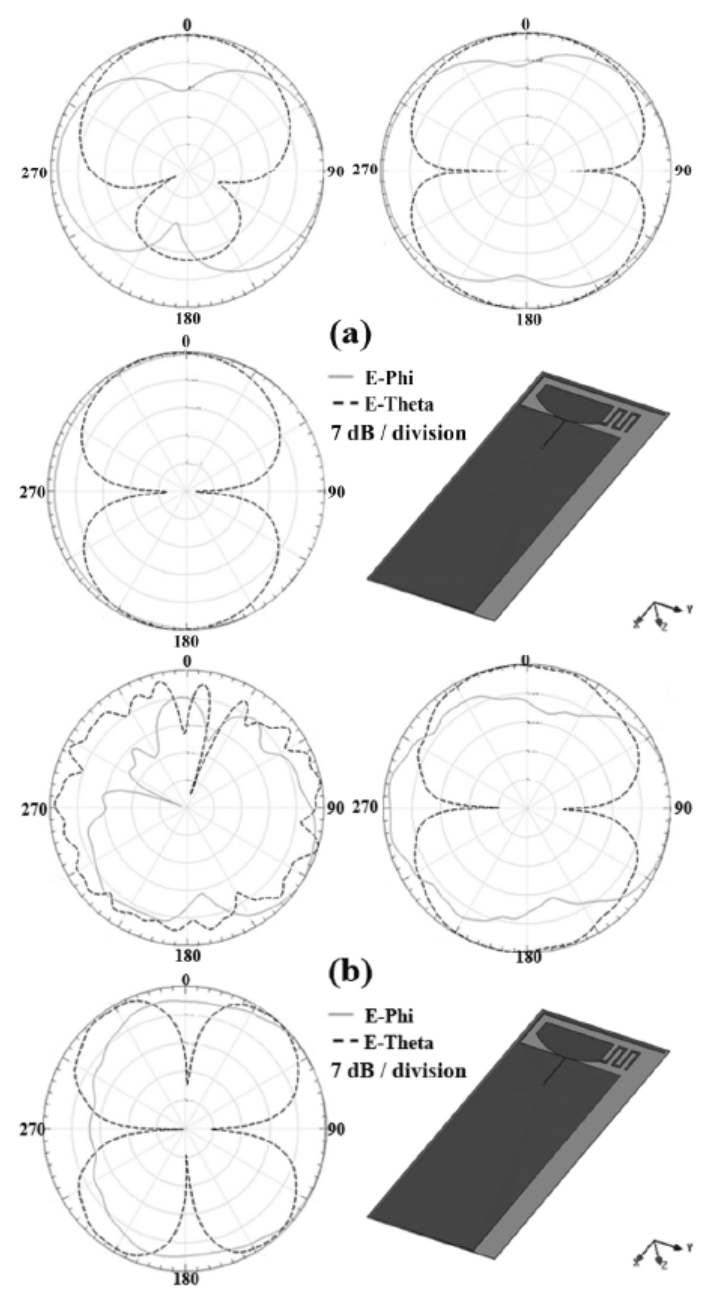

Figure 7: Simulated radiation patterns of the proposed antenna at (a) $0.82 \mathrm{GHz}$, (b) $6 \mathrm{GHz}$. 


\section{References}

[1] Mazinani, S. M. and H. R. Hassani, "A wideband internal plate loaded planar monopole antenna for mobile handset," Journal of Electromagnetic Waves and Applications, Vol. 23, No. 10, 1273-1282, 2009.

[2] Chiu, C. W., C. H. Chang, and Y. J. Chi, "Multiband folded loop antenna for smart phones," Progress In Electromagnetics Research, Vol. 102, 213-226, 2010.

[3] H. Kanj and S.M. Ali, "Compact multiband folded 3-D monopole Antenna,” IEEE Antennas Wireless Propag Lett 8 (2009), 185-188.

[4] C.W. Chi and C.H. Chang, "Multiband folded loop antenna for smart phones,” Prog Electromagn Res PIER 102 (2010), 808-814.

[5] Cheng, P. C., C. Y. D. Sim, and C. H. Lee, "Multi-band printed internal monopole antenna for mobile handset applications," Journal of Electromagnetic Waves and Applications, Vol. 23, No. 13, 1733-1744, 2009.

[6] Wong, K. L., Y. W. Chi, and S. Y. Tu, "Internal multiband printed folded slot antenna for mobile phone application," Microwave Opt. Technol. Lett., Vol. 49, No. 8, 1833-1837, Aug. 2007.

[7] Wu, C. H. and K. L. Wong, "Hexa-band internal printed slot antenna for mobile phone application,” Microwave Opt. Technol. Lett., Vol. 50, No. 1, 35-38, Jan. 2008.

[8] Wong, K.-L., G.-Y. Lee, and T.-W. Chiou, "A low-profile planar monopole antenna for multiband operation of mobile handsets," IEEE Trans. Antennas Propag., Vol. 51, 121-125, Jan. 2003.

[9] Ge, Y., K. P. Essells, and T. S. Bird, "A spiral-shaped printed monopole antenna for mobile communications," IEEE Antennas and Propagation Society International Symposium, 3681-3684, Jul. 2006.

[10] Liu, C.-S., C.-N. Chiu, and S.-M. Deng, "A compact disc-slit monopole antenna for mobile devices," IEEE Antennas Wireless Propag. Lett., Vol. 7, 251-254, 2008.

[11] Wang, H. and M. Zheng, "Triple-band wireless local area network monopole antenna,” IET Microwaves, Antennas \& Propagation, Vol. 2, 367-372, Jun. 2008.

[12] Li, R., B. Pan, J. Laskar, and M. M. Tentzeris, "A compact broadband planar antenna for GPS, DCS-1800, IMT-2000, and WLAN applications," IEEE Antennas Wireless Propag. Lett., Vol. 6, 25-27, 2007.

[13] Zhang, G.-M., J. S. Hong, B.-Z. Wang, Q. Y. Qin, J. B. Mo, and D.-M. Wan, "A novel multi-folded UBW antenna fed by CPW," Journal of Electromagnetic Waves and Applications, Vol. 21, No. 14, 2109-2119, 2007.

[14] Cabedo, A., J. Anguera, C. Picher, M. Ribo, and C. Puente, "Multi-band handset antenna combining a PIFA, slots, and ground plane modes," IEEE Trans. Antennas Propag., Vol. 57, No. 9, 2526-2533, Sep. 2009.

[15] Anguera, J., I. Sanz, J. Mumbru, and C. Puente, "Multi-band handset antenna with a parallel excitation of PIFA and slot radiators," IEEE Trans. Antennas Propag., Vol. 58, No. 2, 348-356, Feb. 2010.

[16] Jing, X., Z. Du, and K. Gong, "A compact multiband planar antenna for mobile handsets," IEEE Antennas Wirel. Propag. Lett., Vol. 5, 343-345, 2006

[17] Sim, C. Y. D., "Mutiband planar antenna design for mobile handset," Microwave Opt. Technol. Lett., Vol. 50, No. 6, 1543-1545, Jun. 2008.

[18] Mahatthanajatuphat, C., S. Saleekaw, and P. Akkaraekthalin, "A Rhombic patch monopole antenna with modified Minkowski fractal geometry for UMTS, WLAN, and mobile WiMAX application," Progress In Electromagnetics Research, Vol. 89, 57-74, 2009.

[19] Jang, B. C. and C. Y. Kim, "Internal antenna design for a triple band using an overlap of return loss," Journal of Electromagnetic Waves and Applications, Vol. 21, No. 8, 1099-1108, 2007.

[20] D.Pozar, "Microwave Engineering," $3^{\text {rd }}$ ed. NewYork Wiley, 2005.

[21] X. Jing, Z. Du, and K. Gong, “A compact multiband planar antenna for mobile handsets," IEEE Antenna Wireless Propag Lett 5 (2006), 343345.

[22] Q. Rao and W. Geyi, "Compact multiband antenna for handheld devices,” IEEE Trans Antennas Propag 57 (2009), 3337-3339.

[23] H. Hsieh, Y. Lee, K. Tiong, and J. Sun, "Design of a multiband antenna for mobile handset operations," IEEE Antenna Wireless Propag Lett 8 (2009), 200-203.

[24] Y. Lee and J. Sun, "A new printed antenna for multiband wireless applications,” IEEE Antenna Wireless Propag Lett 8 (2009), 402-405.

[25] Chia-Ling Liu, Yi-Fang Lin, Chia-Ming Liang,Shan-Cheng Pan, and Hua-Ming Chen, "Miniature Internal Penta-Band Monopole Antenna
forMobile Phone," IEEE TRANSACTIONS ON ANTENNAS AND PROPAGATION, VOL. 58, NO. 3, MARCH 2010.

[26] Owais Owais, Magnus Karlsson, Shaofang Gong,Zhinong Ying, Mathias Grude' n, and Magnus Job, "Wideband planar antenna with modified ground plane,"Microwave Opt. Technol. Lett., vol. 52, No. 11, November 2010 ed.

[27] B.S. Yarman, "Design of Ultra Wideband Antenna Matching Networks".

[28] H. Carrasco, H. D. Hristov, R. Feick, and D. Cofré, "Mutual coupling between planar inverted-F antennas," Microwave Opt. Technol. Lett.,vol. 42, no. 3, pp. 224-227, August 15, 2004.

[29] P.-S. Kildal and K. Rosengren, "Correlation and capacity of MIMO systems and mutual coupling, radiation efficiency and diversity gain of their antennas: Simulations and measurements in reverberation chamber”, IEEE Communications Magazine, vol. 42, no. 12, pp. 102112, Dec. 2004.

[30] K. Rosengren and P. S. Kildal, "Radiation efficiency, correlation, diversity gain and capacity of a six-monopole antenna array for a MIMO system: theory, simulation and measurement in reverberation chamber," Microwaves, Antennas and Propagation, IEE Proceedings -, vol. 152, pp. 7-16, 2005.

[31] J. Carlsson, U. Carlberg, A. Hussain, and P. Kildal, "About Measurements in Reverberation Chamber and Isotropic Reference Environment," in 2010 20th International Conference on Applied Electromagnetics and Communications (ICECom), 20-23 Sept. 2010, Piscataway, NJ, USA, 2010, p. 4 pp.

[32] C. Xiaoming, P. S. Kildal, and J. Carlsson, "Measurement uncertainties of capacities of multi-antenna system in anechoic chamber and reverberation chamber," in Wireless Communication Systems (ISWCS), 2011 8th International Symposium on, 2011, pp. 216-220. 